\title{
Measurement of Femtosecond Electron Bunches
}

D. X. Wang, G. A. Krafft, and C. K. Sinclair

Thomas Jefferson National Accelerator Facility

12000 Jefferson Ave., Newport News, VA 23606

\begin{abstract}
Bunch lengths as short as $84 \mathrm{fs}$ ( $\mathrm{rms}$ ) have been measured at Jefferson Lab using a zero-phasing RF technique. To the best of our knowledge, this is the first accurate bunch length measurement in this regime. In this letter, an analytical approach for computing the longitudinal distribution function and bunch length is described for arbitrary longitudinal and transverse distributions. The measurement results are presented, which are in excellent agreement with numerical simulations.
\end{abstract}




\section{DISCLAIMER}

This report was prepared as an account of work sponsored by an agency of the United States Government. Neither the United States Government nor any agency thereof, nor any of their employees, make any warranty, express or implied, or assumes any legal liability or responsibility for the accuracy, completeness, or usefulness of any information, apparatus, product, or process disclosed, or represents that its use would not infringe privately owned rights. Reference herein to any specific commercial product, process, or service by trade name, trademark, manufacturer, or otherwise does not necessarily constitute or imply its endorsement, recommendation, or favoring by the United States Government or any agency thereof. The views and opinions of authors expressed herein do not necessarily state or reflect those of the United States Government or any agency thereof. 


\section{DISCLAIMER}

Portions of this document may be illegible in electronic image products. Images are produced from the best available original document. 
In recent years, there has been increasing interest in the use of very short electron bunches in short-wavelength FELs, linear colliders, advanced accelerators such as laser or plasma wakefield accelerators, and Compton backscattering X-ray sources [1-3]. The shortest bunch for proposed and existing accelerators is around $100 \mathrm{fs}$ (rms). Bunch length measurement is essential to study beam physics and interaction between the electron beam and the radiation, and to characterize, tune, commission, and operate such shortbunch accelerators. A very stringent demand on final energy spread, with a design goal of $2.5 \times 10^{-5}(\mathrm{rms})$, requires short bunches at the Continuous Electron Beam Accelerator Facility (CEBAF) of Jefferson Lab [4]. CEBAF is routinely operated within its bunch length specification of $0.5 \mathrm{ps}$ (rms), and a bunch length as short as $84 \mathrm{fs}(\mathrm{rms})$ has been achieved. In this letter, the bunch length is defined by the rms width of the longitudinal distribution function.

The conventional technique of measuring short bunches using a streak camera cannot resolve bunch lengths of $100 \mathrm{fs}$ or less [5]. Recently developed frequency domain techniques measuring coherent radiation spectra can only give estimated bunch lengths based on certain assumptions about the longitudinal profile [6-8]. The zero-phasing technique has been used to measure longitudinal profiles and bunch lengths of picosecond bunches. Good consistency was found between measurement and simulations [9-13]. However, the longitudinal profiles reported in Ref. 9 and 11 were obtained where the transverse beam sizes can be neglected, while the formula used in Ref. 10 to calculate bunch lengths is only valid for Gaussian distributions. Furthermore, it is not clear if this technique can be applied to the femtosecond regime. In this letter, analytical formulas are given for computing the longitudinal distribution function and bunch length for arbitrary longitudinal and transverse distributions. Applicability of the technique to femtosecond bunches is demonstrated and confirmed by numerical simulations.

The zero-phasing measurement utilizes several RF accelerating cavities (zerophasing cavities), a spectrometer, and a horizontal profile measuring device. The RF cavities operate at the zero-crossing of the accelerating wave and impart a time-correlated momentum deviation along the beam bunch. Then a spectrometer translates the longitudinal momentum spread into a horizontal position spread. Based on measurement of the horizontal profile, the bunch length and longitudinal distribution function can be determined. 
With the following two assumptions, an analytical expression for the longitudinal distribution function can be derived as a function of the horizontal distribution function and horizontal profile measured at the spectrometer with the zero-phasing cavities on. First, it is assumed that the distribution function in the longitudinal and transverse directions can be separated, i.e.

$$
f\left(x, x^{\prime}, z, z^{\prime}\right)=f_{x}\left(x, x^{\prime}\right) f_{z}\left(z, z^{\prime}\right)
$$

where $f, f_{x}$, and $f_{z}$ are the total, longitudinal, and horizontal normalized distribution functions, respectively. Second, it is assumed that the longitudinal phase space can be treated as an ellipse, and that the intrinsic energy spread or the width of the ellipse can be neglected.

The horizontal position of an electron at the spectrometer can be then written as $x=x_{0}+C z$,where $x_{0}$ is the nondispersive part,

$$
C=C_{0}+C_{1}=\left(2 \pi \cdot e V_{r f} / \lambda_{r f}+d E / d z\right) \cdot \eta / E_{0},
$$

$\eta$ is dispersion of the spectrometer, $E_{0}$ is average beam energy, $\lambda_{\text {rf }}$ is the RF wavelength, $V_{r f}$ is the summed accelerating voltage of zero-phasing cavities, and $d E / d z$ is the slope of the longitudinal phase space ellipse. The linear slope of the accelerating voltage of the zero-phasing cavities, i.e. $2 \pi \cdot e V_{r f} / \lambda_{r f}$, is used under the approximation that the RF wavelength is much longer than the bunch length. The horizontal profile measured at the spectrometer with the zero-phasing cavities on is then found to be

$$
F(x)=\iint f_{x}\left(x, x^{\prime}\right) d x^{\prime} f_{z}\left(z, z^{\prime}\right) d z d z^{\prime}=\int f_{x}\left(x_{0}+C z\right) f_{z}(z) d z \text {. }
$$

where. $\int f_{x}\left(x, x^{\prime}\right) d x^{\prime}=f_{x}(x)$ and $\int f_{z}\left(z, z^{\prime}\right) d z^{\prime}=f_{z}(z)$ are used by definition. Therefore, the measured horizontal profile is simply a convolution of the longitudinal distribution function and the horizontal distribution function. The longitudinal distribution function can be deconvolved as

$$
f_{z}(z)=\int\left[\frac{\int F(x) e^{i k x} d x}{\int f_{x}(x) e^{i k x} d x}\right] e^{i C z k} d k .
$$

Now $f_{x}(x)$ may be directly measured with an experiment arrangement where the profile measurement device is placed in a straight beam line at the same distance from the dipole and with the same optical properties as the one at the spectrometer. $f_{x}(x)$ may be approximated by the horizontal profile measured at the spectrometer with the zero-phasing 
cavities off, if the dispersive part of the initial energy spread is small compared to the horizontal beam size. Such an approximation was made for the following measurement. If the horizontal distribution function and the measured horizontal profiles are Gaussian, the longitudinal distribution function is also Gaussian. From Eq. (4), a relation between longitudinal bunch length and measured transverse bunch widths is found to be

$$
\sigma_{z}=\left(\Omega^{2}-\sigma_{x}^{2}\right)^{1 / 2} / C
$$

where $\sigma_{z}, \Omega$, and $\sigma_{x}$ are corresponding widths of the Gaussian profiles, as used in Ref. 8. If $f_{x}(x)$ is negligibly small compared to $F(x)$ and can be replaced by a delta function, the longitudinal distribution function equals the measured profile, i.e. $f_{z}(z)=F(z=x / C)$ as expected, with a scale conversion factor $C$ between the horizontal and longitudinal dimensions. Usually, $C_{0}$ can be experimentally determined but $d E / d z$ or $C_{1}$ is unknown. To overcome this problem, the horizontal profiles are measured at both zerocrossing points of the RF waves, which alters the sign of $C_{0}$. It is noticed that using $C_{0}$ instead of $C$ in Eq. (4) is equivalent to compressing the $z$ axis by a factor of $C / C_{0}$. Therefore,

$$
z_{\text {rms }}=z_{m s}^{+} \cdot C_{0} /\left(C_{0}+C_{1}\right)=z_{m i s}^{-} \cdot C_{0} /\left(C_{0}-C_{1}\right)
$$

where $z_{r m s}$ is the longitudinal rms width calculated from the distribution function derived from Eq. (4) using $C$, and $z_{r m s}^{+}$and $z_{r m s}^{-}$are the corresponding rms widths using $C_{0}$ when $C_{0}$ and $C_{1}$ are the same and opposite signs, respectively. Then the rms width and initial phase space slope normalized by the RF slope are given by

$$
z_{r m s}=\left(z_{r m s}^{+}+z_{r m s}^{-}\right) / 2
$$

and

$$
C_{1} / C_{0}=(d E / d z) /\left(2 \pi \cdot e V_{r f} / \lambda_{r f}\right)=\left(z_{r m s}^{+}-z_{r m s}\right) / z_{r m s}
$$

Accordingly, the $z$ axis for both measured profiles needs to be corrected by the corresponding factor of $C_{0} / C$. The difference between the two resulting distribution functions indicates the validation of the assumptions. It is worth mentioning that the above calculation may also be applied to other similar measurements such as deconvoluting the longitudinal profile from streak camera measurements, though the same assumptions are required. 
The formula and measurement procedure were tested using PARMELA simulation, which has been used extensively during CEBAF commissioning to verify experimental results. Very good agreement has been found between various measurements and simulation results in the past. The zero-phasing measurement was performed numerically and compared to the actual bunch length. The test case showed good agreement over bunch lengths in the range of 100 to $400 \mathrm{fs}$, with a $10 \mathrm{fs}$ systematic offset, indicating that the assumptions are good approximations.

The measurement was carried out at the CEBAF injector. A block diagram of the injector layout is given in Fig. 1. A $100 \mathrm{keV} \mathrm{CW}$ electron beam is generated and chopped by a pair of RF chopper cavities into a bunch train with variable duration from 0 to $40 \mathrm{ps}$ (rms) separated by $2 \mathrm{~ns}$. The beam is bunched by an RF buncher and accelerated to 500 $\mathrm{keV}$ by an RF "capture" cavity, a 5 cell variable $\beta$ normal RF accelerating structure. Then the beam is further bunched and accelerated to $5 \mathrm{MeV}$ by the two superconducting RF (SRF) cavities. Following acceleration to the final injection energy of $45 \mathrm{MeV}$ by $16 \mathrm{SRF}$ cavities, little additional bunching occurs, due to longitudinal relativistic effects. Nominally, 16 SRF cavities in the first and second SRF modules run on crest to achieve maximum energy gain and minimum energy spread. During the measurement of the shortest bunches, the last 8 SRF cavities are phased to plus and minus $90^{\circ}$ off crest. A wire scanner is used to measure horizontal profile at the spectrometer. Three typical profiles are shown in Fig. 2 and can be fit closely to Gaussian distributions. Both zero phases of each individual zero-phasing cavity are determined by finding the phase that yields zero transverse movement at the spectrometer viewer when the cavity is turned on and off. The beam energy and summation of the zero-phasing cavity gradient were measured by the spectrometer Hall probe. The experimental parameters in this measurement are: $\lambda_{r f}$ of $20 \mathrm{~cm}, \eta$ of $1.52 \mathrm{~m}, V_{r f}$ of about $20 \mathrm{MV}$, and $E_{0}$ of about 25 $\mathrm{MeV}$. The bunch length was systematically changed by varying the second SRF cavity phase, resulting in a serial longitudinal phase space rotation. Excellent agreement has been achieved between the measurement and simulation, shown in Fig. 3. In addition, the measurement results are consistent with a power measurement of the coherent synchrotron radiation (CSR). As expected, CSR power increased when the bunch length became shorter, and minimum bunch length yielded maximum CSR power [14]. The left side of Eq. (8) is plotted from simulation in the solid line while the right side is displayed from measurement in the circles of Fig. 4, as the phase of the second SRF bunching cavity is varied as in Fig. 3. Measurements and simulation results agree well. It is noted that the zero value point represents the upright position of the ellipse in the longitudinal phase 
space, where the shortest bunch was obtained in both experiment and simulation, i.e. the minimum point in Fig. 3. There is a steep slope around the zero point where the slope of the ellipse changes sign, corresponding to the transition from undercompression to overcompression. Therefore, the maximum slope of the ellipse $d E / d z$ is about a factor of seven smaller than the RF slope, $2 \pi \cdot e V_{r f} / \lambda_{r f}$ of $720 \mathrm{MeV} / \mathrm{m}$.

The zero-phasing technique provides a means of measuring the longitudinal density distribution function and the bunch length of fs bunches. Its resolution is mainly limited by the constraints of the specific machine configuration. In practice the following considerations may be given to better interpret the measurement results: (1) measure the horizontal distribution function at the straight-ahead location and the horizontal profile at the spectrometer with the zero-phasing cavities off, which provides information about the longitudinal phase space ellipse, with orientation information obtained from Eq. (8); (2) focus the beam spot tightly in the horizontal direction; (3) increase the energy spread produced by the zero-phasing cavities. (4) the errors due to RF amplitude and phase stability can be estimated from horizontal beam jitters at the spectrometer viewer, which result in a longer measured bunch length. Similarly to a streak camera, a bunch-to-bunch resolution may be obtained by using a vertical RF sweeper after the spectrometer dipole plus a gated camera. The zero-phasing technique has been proven to be crucial at CEBAF for characterizing the bunching process and calibrating a noninvasive CSR bunch length monitor that is an invaluable tool for machine operations.

In summary, an electron bunch length as short as $84 \mathrm{fs}$ has been measured using the zero-phasing technique. Analytical formulas for calculating the longitudinal distribution function and bunch length are derived. To the best of our knowledge, this is the first accurate bunch length measurement in the regime of less than $100 \mathrm{fs}$, and the zero-phasing is the only technique that has demonstrated such a capability. Numerical simulation provides validation of the assumptions of the technique. The systematic measurement results are in excellent agreement with the simulation and consistent with CSR power measurements.

The authors would like to thank B. Dunham and H. Liu for help running the PARMELA simulation code and CEBAF operations personnel for help on the measurements. This work was supported by U.S. DOE under contract \#DE-AC05-84ER40150. 
References:

[1] H. Winick, et al., Nucl. Instrum. Methods A347 (1994), 199.

[2] R. H. Sieman, Proceedings of the 1993 Particle Accelerator Conference, Washington, D. C., May 17-20, 1993, 532.

[3] W. B. Mori, et al., AIP Conference Proceedings 335, 112 (1994).

[4] G. A. Krafft, Proceedings of the 1994 International Linear Accelerator Conference, Tsukuba, Japan, August 21-26, 1994, 9.

[5] A. Lumpkin, AIP Conference Proceedings 367, 327 (1995).

[6] K. Ishi, et al., Phys. Rev. A 43 \#10, 5597 (1991).

[7] P. Kung, et al., Phys. Rev. Lett. 73 \#7, (1994).

[8] H. Lihn, et al., Phys. Rev. E 53 \#6, 6413, (1996).

[9] L. Rivkin et al., Proceedings of the 1st European Particle Accelerator Conference, Rome, Italy, June 7-11, 1988, 634.

[10] J. Seeman et al., Proceedings of the 1988 International Linear Accelerator Conference, Newport News, VA, October 3-7, 1988, 441.

[11] K. Bane, SLAC-PUB-5177, Feb. 1990 (A).

[12] K. Bane et al., Proceedings of the 2nd European Particle Accelerator Conference, Nice, France, June 12-16, 1990, 1762.

[13] K. Bane et al., SLAC/AP-80, Sept. 1990 (AP).

[14] D. X. Wang et al., Appl. Phys. Lett. 70 \#4, 529, (1997). 
Figure captions:

Fig. 1. Schematic block diagram of CEBAF injector layout.

Fig. 2 Horizontal profile measured by the wire scanner at the spectrometer, where the scanner signal is proportional to the charge density and the circles are from measurement and the solid curves are Gaussian fittings. Profile (a) was measured with the zero-phasing cavities off while profiles (b) and (c) were measured with the zero-phasing cavities at $+90^{\circ}$ and $-90^{\circ}$ off the crest.

Fig. 3 The bunch length versus the second SRF cavity phase, where the circles are from measurement and the solid curve is from simulation.

Fig. 4 The normalized longitudinal phase space slopes. The circles are the measurement of the right-hand side of Eq. 8, $\left(z_{m m s}^{+}-z_{r m s}\right) / z_{r m s}$, while the solid curve is the left -hand side, $(d E / d z) /\left(2 \pi \cdot e V_{r f} / \lambda_{r f}\right)$, from simulation. 


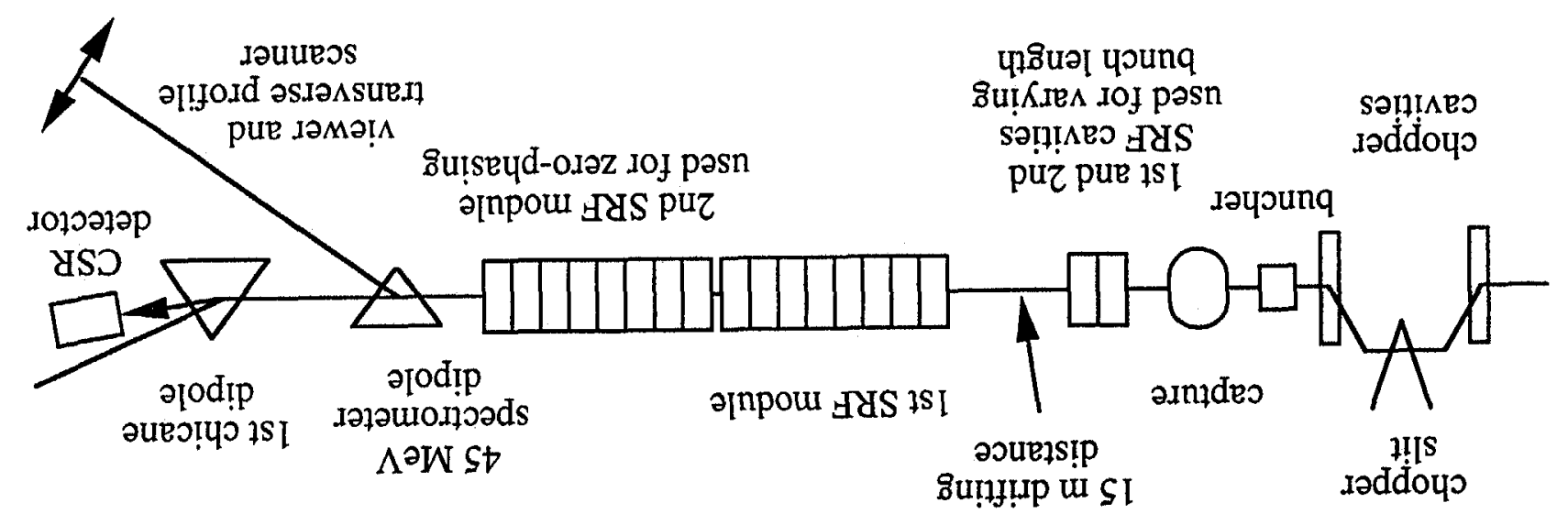



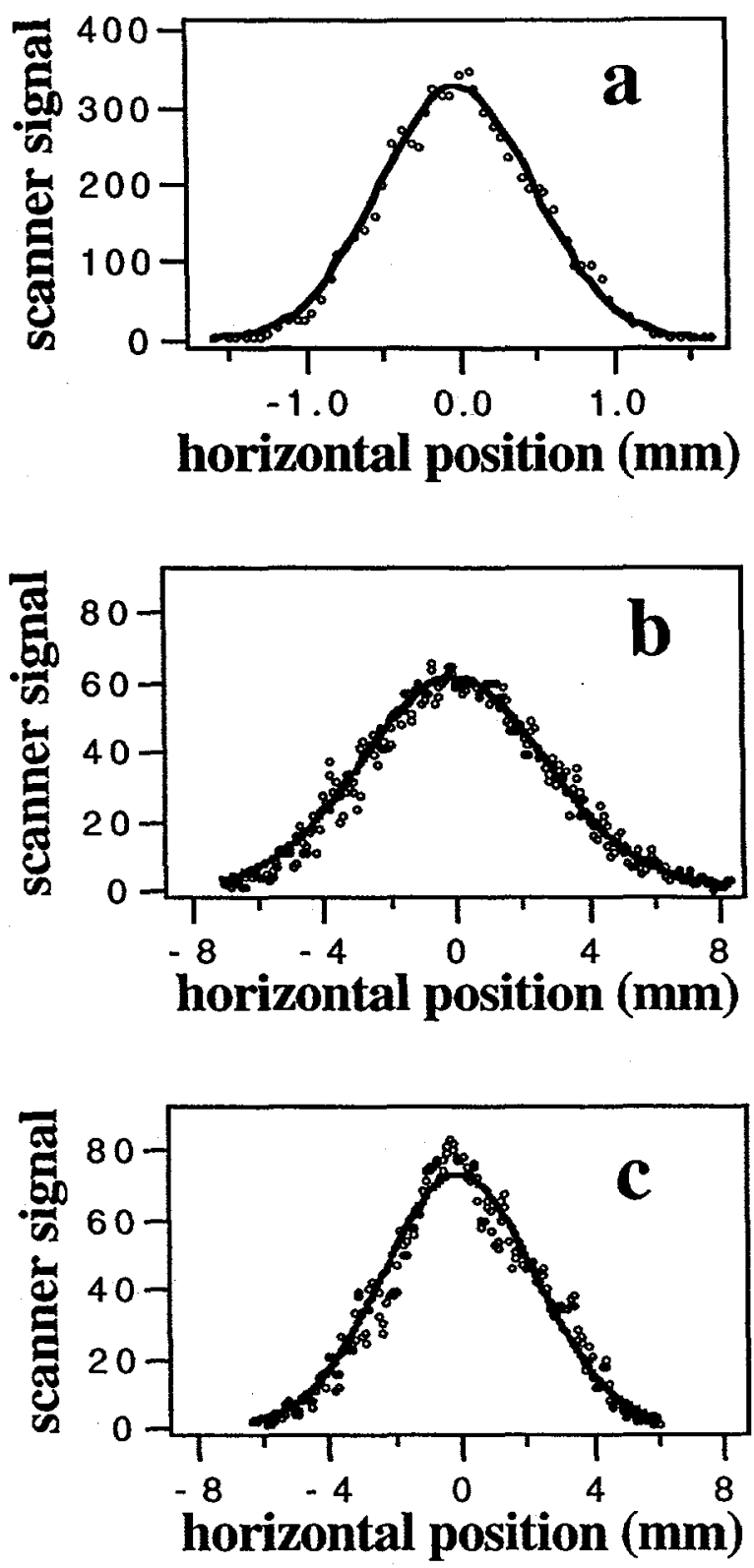


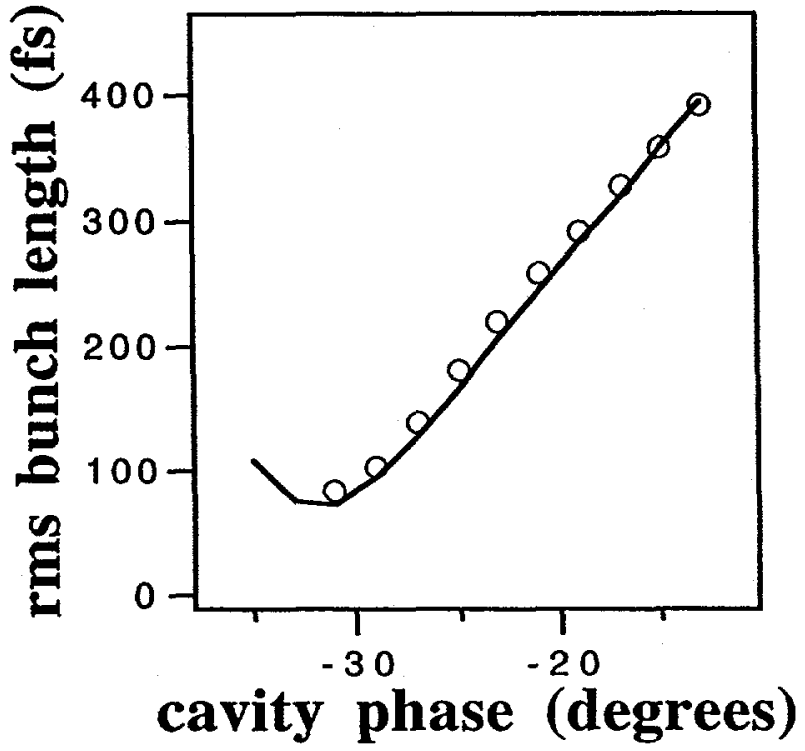




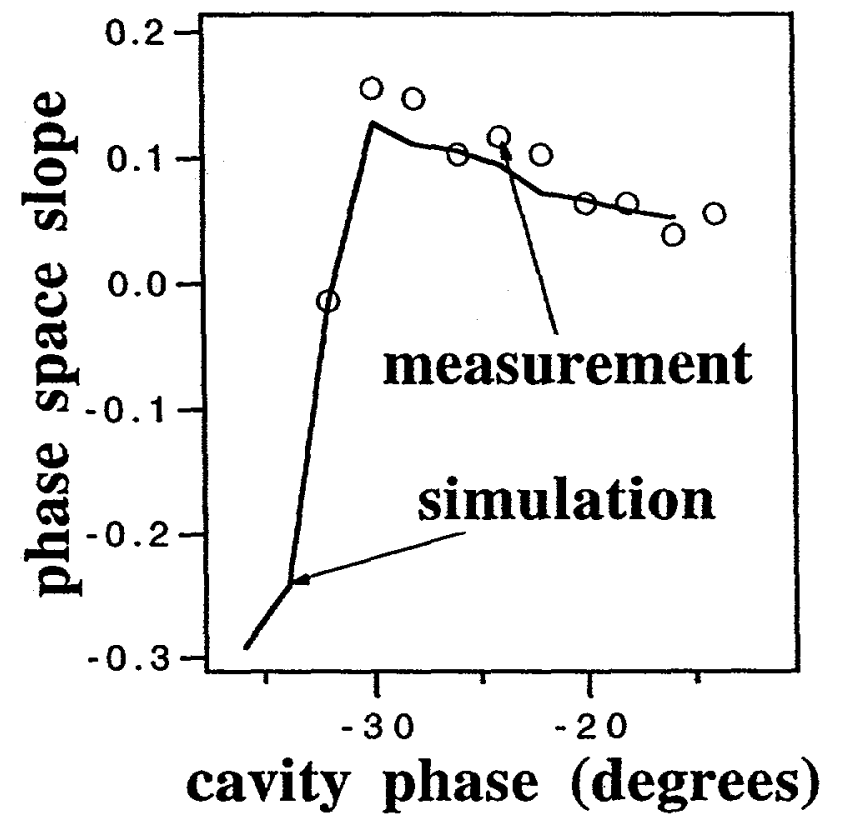

